\title{
X-RAY CHARACTERIZATION OF GaAs:Zn GAS-TRANSPORT GROWN WHISKERS USING CONVENTIONAL AND SYNCHROTRON SOURCES
}

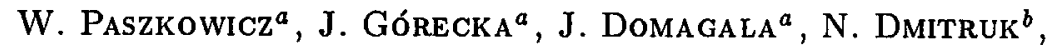 \\ S.S. Varshava ${ }^{c}$, J. Härtwig ${ }^{d}$, M. OhleR ${ }^{d}$ and A. Pietraszko ${ }^{e}$ \\ a Institute of Physics, Polish Academy of Sciences \\ Al. Lotników 32/46, 02-668 Warsaw, Poland \\ ${ }^{b}$ Institute for Physics of Semiconductors, National Academy of Sciences, Kiev, Ukraine \\ ${ }^{c}$ State University of Technology, 290648 Lviv, Ukraine \\ ${ }^{d}$ European Synchrotron Radiation Facility, 38043 Grenoble, France \\ ${ }^{\mathbf{e}}$ Institute of Low Temperatures and Structural Research, Okólna 2, Wrocław, Poland
}

\begin{abstract}
GaAs:Zn whiskers grown by the gas-transport method are characterized by diffraction methods using white and monochromatic radiation. The methods applied include the white-beam topography at ESRF synchrotron source and Laue patterns, 4-circle Bond diffractometry and high-resolution diffractometry at conventional X-ray sources. The results obtained concern the growth morphology and defect structure. It is found that GaAs:Zn whiskers grown by the described method have the form of long needles and blades of the morphologies represented by growth direction and largest lateral face $\langle 112\rangle\{111\}$ and $\langle 111\rangle\{112\}$, respectively, with a single exception of a blade of uncommon morphology $\langle 111\rangle\{110\}$.
\end{abstract}

PACS numbers: $61.72 . \mathrm{Dd}, 68.70 .+\mathrm{w}$

\section{Introduction}

GaAs is one of technologically important materials. Its physical properties strongly depend on the doping level and defect structure. Investigation of various growth methods is of high interest because each of them may introduce specific impurities and structural defects and thus it may influence the properties of the crystals.

So-called whiskers, i.e. natural or synthetic single crystals of thread- and ribbon-like shape, exhibit interesting physical properties. General information about the growth and properties of such crystals may be found, e.g., in Refs. [1-3]. They adopt various morphologies which include long threads and tapes, thin platelets, tubular and spiral, cone- and wedge-shaped crystals, etc. Whiskers are characterized by strong dimensional disproportions (reaching even several orders 
of magnitude) along different crystallographic directions. For example, a platelet length may be thousand times larger than the thickness. In most cases the lateral faces are crystallographic planes of low Miller indices. The range of typical whisker thickness is $0.01-100 \mu \mathrm{m}$, the length may attain several centimeters [4]. Among reported whiskers, those formed from semiconducting materials constitute an important part. For example, in Ref. [5] the growth of $\mathrm{Si}$ whiskers of length $15 \mathrm{~mm}$ and diameter $0.3 \mathrm{~mm}$ is described. In Ref. [6] $\mathrm{In}_{2} \mathrm{Te}_{3}$ whiskers of about $0.1 \mathrm{~mm}$ diameter have been reported. In specific conditions dendrite-like whiskers are formed (see [7] for GaAs and GaP crystals grown from gas phase). In Ref. [8] it is indicated that such dendrites contain two or more twin boundaries. For free-standing $\mathrm{Si}_{1-x} \mathrm{Ge}_{x}$ filament crystals a decrease in surface defect concentration, an increase in crystal strength and a dependence of lattice parameter on whisker diameter have been concluded [9]. GaAs whiskers of various shape (stubs, blades, triangular, hexagonal and twelve-sided needles) have been described in the literature, see for example [10-13]. Possible applications of GaAs whiskers include strain and temperature sensors [14]. One of the interesting structural properties of micrometer-size GaAs whiskers is the possibility of adopting an (untypical for GaAs) polytype structure type, namely, wurtzite $[10,11]$ or $4 \mathrm{H}[10]$ (thin layers of wurtzite type GaAs have also been reported [15]).

\section{Experimental}

The GaAs:Zn whiskers were grown at Lviv University of Technology by the gas-transport method in the systems $\mathrm{Ga}-\mathrm{As}-\mathrm{ZnCl}_{2}$ and $\mathrm{GaAs}-\mathrm{ZnCl}_{2}$. A relation between the mass transport rate in these systems and the growth rate was studied. The growth conditions were selected on the basis of both the thermodynamical analysis of vapour-transport systems and a preliminary study on the influence of growth parameters on whisker quality. GaAs: $\mathrm{Zn}$ polycrystals were synthesised in the system $\mathrm{Ga}-\mathrm{As}-\mathrm{ZnCl}_{2}$ in the temperature between 800 and $1100^{\circ} \mathrm{C}$. Then, in the same ampoule at the temperature 900 to $1000^{\circ} \mathrm{C}$ the $\mathrm{GaAs}: \mathrm{Zn}$ whiskers were grown. The final cycle time was 3 hours. Optimum $\mathrm{ZnCl}_{2}$ concentration was $c_{\mathrm{Zn}}=0.5$ to $1.5 \mathrm{mg} / \mathrm{cm}^{3}$. For smaller $c_{\mathrm{Zn}}$, the mass transport of $\mathrm{GaAs}$ is weak, while for $c_{\mathrm{Zn}}>2 \mathrm{mg} / \mathrm{cm}^{3}$ the defect density increases and dendritic growth is observed. The maximum length of the crystals grown with the described method may attain about $4 \mathrm{~cm}$. The crystals characterized in the present work were of 5-10 mm length, thicknesses of the order of 10 microns and from several tens to $400 \mu \mathrm{m}$ width of lateral face.

The characterization methods applied include:

- white-beam topography at ESRF synchrotron source,

- Laue patterns, Bond diffractometry and high-resolution diffractometry at conventional laboratory sources.

A high-resolution diffractometer, Philips MRD, was applied for measurement of rocking curves and for reciprocal space mapping as well as for preliminary evaluation of the lattice constant. The measurement conditions at MRD were as follows: $1 \mathrm{~mm}$ vertical slit, Ge-220 channel-cut 4-reflection monochromator and (for reciprocal space maps) a similar 2-reflection channel-cut analyzer crystal. For 
measurements with this diffractometer, the $\mathrm{Cu} K_{\alpha_{1}}$ radiation was applied $(\lambda=$ $1.540598 \AA$ ),

Precise measurements of lattice constant by the Bond method were performed using KM4B, a new type of commercial fully automatic 4-circle diffractometer [16]. This facility is suitable for the precise measurement of the lattice constants of small crystals. It is equipped with a graphite monochromator at the incident beam and with a long collimator. $\mathrm{Cu} K_{\alpha}$ radiation $(\lambda=1.540562 \AA)$ was used. High-angle reflections $-1,-1,7,-7,-1,1,-1,-7,1$ were measured, each of them 6 times. The absolute value of the lattice constant was calibrated with the help of a standard silicon crystal.

The advantages of $\mathrm{X}$-ray diffraction topography at third-generation synchrotron radiation facilities are well known [17], in particular high resolution (several microns) investigation of very small crystals becomes possible in such conditions. The defect structure was investigated by the white-beam topography at ESRF synchrotron source and at a high-resolution Philips MRD laboratory diffractometer.

\section{Results and discussion}

The results obtained concern the growth direction and orientation of external faces, morphology, defect structure and resistivity. The topographs of all nine crystals show that the degree of perfection is a property of the given whisker and no correlation betwecri the zinc content, the whisker shape (observed at an optical microsope) nor size could be found. For two crystals, which are extreme cases of kinds of defects, N111 and P117, rocking curves and reciprocal space maps at 111
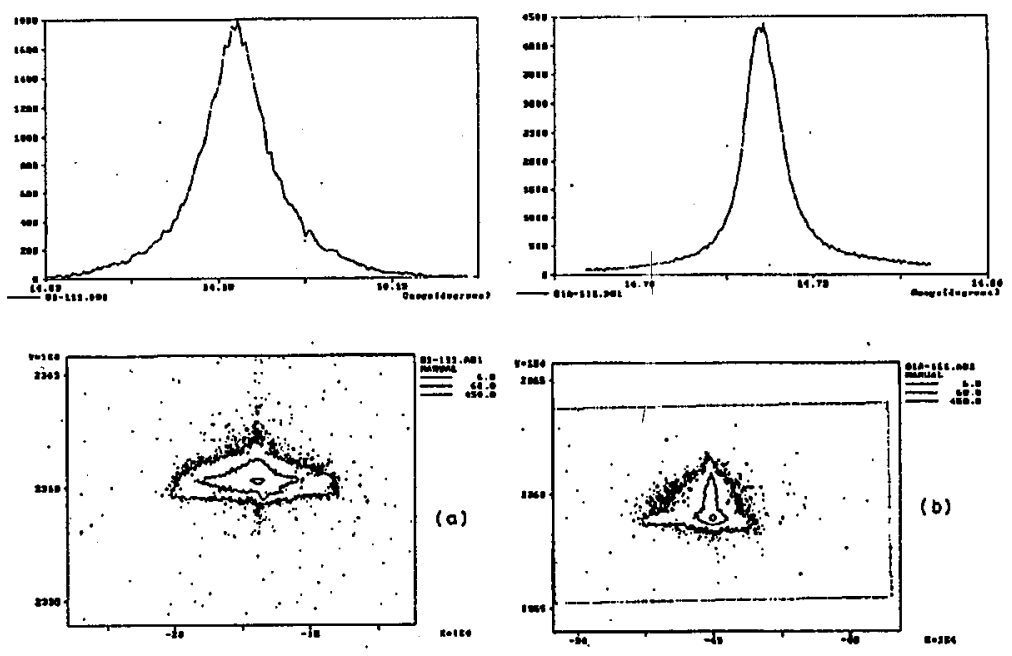

Fig. 1. Rocking curves (upper figures) and reciprocal space maps (lower figures) for samples N11 and P117 obtained at the 111 lateral face using 111 reflection: (a) N111 (FWHM $=68$ arcsec), (b) P117 (FWHM $=45$ arcsec). The intensity at contours at each reciprocal space map increases towards the figure center. 

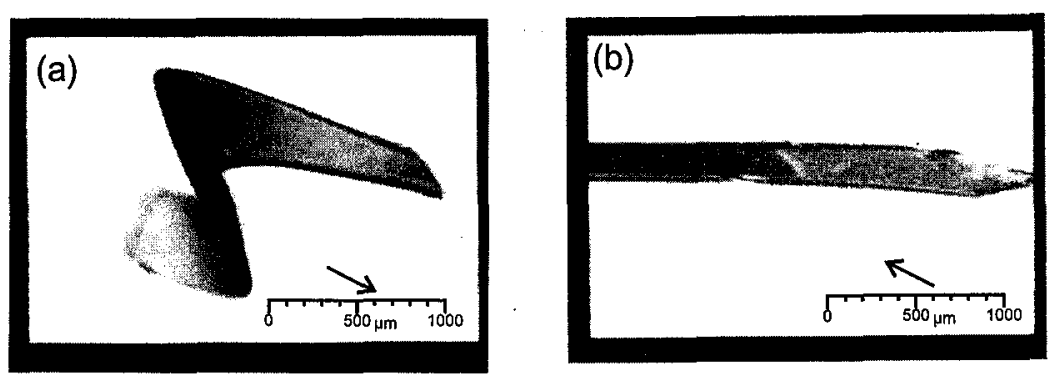

Fig. 2. Topographs for samples N111 (a) and P117 (b). Arrows refer to the direction of the diffraction vector.

\section{TABLE I}

Lattice constant of an intentionally undoped GaAs whisker of about $10 \mathrm{~mm}$ in length. Measurements were performed at $26.8^{\circ} \mathrm{C}$ on $\mathrm{KM} 4 \mathrm{~B}$ diffractometer.

\begin{tabular}{c|c|c}
\hline \hline Location & Reflection & $\begin{array}{c}\text { Lattice parameter } \\
{[\AA]}\end{array}$ \\
\hline \multirow{2}{*}{ one end } & $-1,-1,7$ & $5.65335(2)$ \\
& $-7,-1,1$ & $5.65335(2)$ \\
& $-1,-7,1$ & $5.65335(2)$ \\
\cline { 3 - 3 } & & $5.65335(1)$ (mean) \\
\hline \multirow{2}{*}{ second end } & $-1,-1,7$ & $5.65331(2)$ \\
& $-7,-1,1$ & $5.65343(2)$ \\
& $-1,-7,1$ & $5.65331(2)$ \\
\cline { 2 - 3 } & & $5.65335(1)$ (mean)
\end{tabular}

reflection enabled evaluation of the structural quality (Fig. 1). The following results were obtained: for N111 the FWHM is 68 arcsec while for P117 it is $45 \operatorname{arcsec}$. A reason of this difference was found at topographs (Fig. 2) and reciprocal space maps: a higher FWIIM for N111 is explained by a curvature of crystallographic planes seen on the topograph and by the reciprocal space map, where a broadening perpendicular to the diffraction vector occurs indicating the presence of curvature (there is no such strong broadening effect for P117). A preliminary measurement of lattice constant at MRD diffractometer using a low-angle reflection indicates that for a higher $\mathrm{Zn}$ content (sample P117, $\rho=0.01 \Omega \mathrm{cm}$ ) the lattice constant is larger than for a lower $\mathrm{Zn}$ content (sample N111, $\rho=0.1 \Omega \mathrm{cm}$ ), in agreement with the reported influence of $\mathrm{Zn}$ doping causing an increase in lattice parameter of GaAs [18]. The localized defects appearing on the topograph of P117 are not due to lattice-constant local variation, because there is no broadening parallel to the diffraction vector relative to N111. For one intentionally undoped crystal the lattice constant was measured hy the Bond method. The results obtained (Table I) show with an accuracy of $10^{-5} \AA$ that the lattice parameter does not change 
TABLE II

Morphology of undoped ( $n$-type, denoted by the " $\mathrm{N}$ " letter in the samples symbol) and Zn-doped ( $p$-type, denoted by "P") GaAs:Zn whiskers grown by gas-transport method. Literature data are given for comparison at the end of the table. Shape symbols: $\mathrm{B}$ - blade, $\mathrm{T}$ - needle of triangular section. Abbreviations for observed defects: c - curvature of lattice planes, (c) - weak curvature, lc - localized strong curvature, $\mathrm{d}-$ localized microdefects in the whole crystal, id - isolated domains of microdefects, $\mathbf{d}$ ? - presence of defects not seen due to insufficient resolution at the given topograph. For samples P117 and P3B the whisker width varies smoothly along the crystal.

\begin{tabular}{|c|c|c|c|c|c|c|}
\hline \multirow[t]{2}{*}{ Sample } & \multicolumn{3}{|c|}{ Description of crystal } & \multirow[t]{2}{*}{ Growth axis } & \multirow{2}{*}{$\begin{array}{c}\text { Measured } \\
\text { lateral face }\end{array}$} & \multirow{2}{*}{$\begin{array}{r}\rho \\
{[\Omega \mathrm{cm}]}\end{array}$} \\
\hline & Shape & $\begin{array}{c}\text { Width } \\
{[\mu \mathrm{m}]}\end{array}$ & Defects & & & \\
\hline P117 & B & $80-230$ & $\mathrm{~d}$ & $\langle 112\rangle$ & $\{111\}$ & 0.01 \\
\hline N111 & B & $370-430$ & c & $\langle 112\rangle$ & $\{111\}$ & 0.1 \\
\hline P89 & B & 160 & lc,id & $\langle 112\rangle$ & $\{111\}$ & 0.009 \\
\hline P133 & B & 90 & lc,d? & $\langle 112\rangle$ & $\{111\}$ & 0.024 \\
\hline $\mathrm{P} 1 / 1$ & B & $100-140$ & (c), d? & $\langle 111\rangle$ & $\{110\}$ & 0.001 \\
\hline P116 & B & 150 & c & $\langle 112\rangle$ & $\{111\}$ & 0.02 \\
\hline P135 & $\mathrm{B}$ & 120 & lc,id & $\langle 112\rangle$ & $\{111\}$ & 0.019 \\
\hline P91 & $\bar{T}$ & 150 & (c), d & $\langle 111\rangle$ & $\{112\}$ & 0.135 \\
\hline P3B & $\mathrm{T}$ & $25-170$ & (c), d? & $\langle 111\rangle$ & $\{112\}$ & 0.021 \\
\hline $\mathrm{GaAs}[12]$ & \multicolumn{3}{|l|}{ blade } & $\langle 001\rangle$ & \multicolumn{2}{|l|}{110} \\
\hline GaAs [9] & \multicolumn{3}{|c|}{ large blade } & $\langle 112\rangle$ & \multicolumn{2}{|l|}{111} \\
\hline GaAs [13] & \multicolumn{3}{|c|}{ twinned blade } & $\langle 112\rangle$ & \multicolumn{2}{|l|}{111}$\{113\}$ \\
\hline GaAs [12] & \multicolumn{3}{|c|}{ needle/blade } & $\langle 112\rangle$ & \multicolumn{2}{|c|}{111}$\{110\}\{113\}^{*}$ \\
\hline $\mathrm{GaAs}$ [12] & \multicolumn{3}{|l|}{ needle } & $\langle 111\rangle$ & \multicolumn{2}{|l|}{110}$\{112\}^{*}$ \\
\hline GaAs $[10]$ & \multicolumn{3}{|l|}{ needle ${ }^{\dagger}$} & $\langle 111\rangle$ & \\
\hline GaAs [13] & \multicolumn{3}{|l|}{ needle } & $\langle 111\rangle$ & & $\{112\}$ \\
\hline GaAs [13] & \multicolumn{3}{|c|}{ trigonal prism } & & \multicolumn{2}{|l|}{112} \\
\hline
\end{tabular}

*possible lateral faces of various crystals,

$t$ possibility of formation of two-layer (wurtzite) and four-layer (4II) polytypes in micrometer size whiskers has been reported in Ref. [10].

along the crystal. A more detailed investigation of the lattice parameter behaviour for doped and undoped GaAs whiskers is anticipated. Laue diffraction patterns show that most frequently the $\mathrm{Zn}$ doped GaAs whiskers grown by the described method have the form of blades and needles, the growth direction and orientation of the largest external faces being (see Table II) $\langle 112\rangle\{111\}$ and $\langle 111\rangle\{110\}$ for the blades of rectangular section, $\langle 111\rangle\{112\}$ for the needles of triangular section. The morphology of the crystals studied generally agrees with the cited literature data $[9,10,12,13]$ in tendencies of blades and needles to adopt the mentioned morphologies, except for sample P1/1, where the lateral face is $\{110\}$. 


\section{Acknowledgments}

The Philips MRD diffractometer was sponsored by the Foundation for Polish Science within the program SEZAM94. The authors are indebted to Dr D. Kucharczyk (Institute of Low Temperatures and Structural Research) for the participation in measurements. The partial support by the Committee for Scientific Research grant No. 101/P3/93/05 and Polish-French Cooperation Joint Project No 5197/1995 are acknowledged.

\section{References}

[1] Metallurgy of Elemental and Compound Semiconductors, Ed. R.O. Grubel, Interscience, New York 1961.

[2] The Art and Science of Growing Crystals, Ed. J.J. Gilman, John Wiley \& Sons, New York 1965.

[3] G.V. Berezhkova, Nitevidnye kristally (Thread-like crystals), Nauka, Moscow 1969 (in Russian).

[4] E.I. Givargizov, Rost nitevidnykh i plastinchatykh kristallov iz para (Growth of Thread-like and Plate-like Crystals Form Vapour), Nauka, Moscow 1977 (in Russian).

[5] R. Wagner, in: Monokristal'nye volokna i armirovannye nimi materialy, Mir, Moscow 1973, p. 77 (Russian translation).

[6] A.G. Kunjomana, E. Mathai, Mater. Res. Bull. 26, 1347 (1991).

[7] H.-J. Dersin, E. Sirtl, Z. Metallkunde 55, 536 (1964).

[8] W. Krieglstein, H. Pfister, G. Ziegler, Z. Angew. Phys. 17, 295 (1964).

[9] A.I. Klimovskaya, I.P. Ostrovskii, R.I. Baitsar, A.S. Ostrovskaya, J. Phys., Condens. Matter 7, 1229 (1995).

[10] M. Koguchi, H. Kakibayashi, M. Yazawa, K. Hiruma, T. Katsuyama, Jpn. J. Appl. Phys. 31, 2061 (1992).

[11] E. Laverko, V.M. Makharonov, S.M. Polyakov, Kristallografiya 10, 732 (1965).

[12] R.L. Barns, W.C. Ellis, J. Appl. Phys. 36, 2296 (1965).

[13] A.V. Sandulova, G.F. Mocharnyuk, K.S. Shcherbai, S.S. Varshava, R.N. Skoropad, Izv. AN SSSR 8, 945 (1972).

[14] S.S. Varshava, G.A. Kitin, A.A. Manenkov, G.I. Mikhailova, V.F. Troitskii, K.S. Shcherbai, Prib. Tekh. Eksp. 1, 219 (1987).

[15] E.K. Müller, J. Appl. Phys. 35, 580 (1964).

[16] D. Kucharczyk, A. Pietraszko, K. Łukaszewicz, J. Appl. Crystallogr. 26, 467 (1993).

[17] R. Barrett, J. Baruchel, J. Härtwig, F. Zontone, J. Phys. D, Appl. Phys. 28, 4A, A250 (1995).

[18] V.F. Stelmakh, A.R. Chelyadinskii, Elektrotekhnika, set 6: Materialy 104, 52 (1977). 